九州大学学術情報リポジトリ

Kyushu University Institutional Repository

\title{
URYSOHN'S LEMMA IN SCHRÖDER CATEGORIES
}

Kawahara, Yasuo

Department of Informatics, Kyushu University

https://doi.org/10.5109/16775

出版情報: Bulletin of informatics and cybernetics. 39, pp.69-81，2007-12. Research Association of Statistical Sciences

バージョン :

権利関係 : 


\title{
URYSOHN'S LEMMA IN SCHRÖDER CATEGORIES
}

\author{
by
}

Yasuo KaWAHARA

Reprinted from the Bulletin of Informatics and Cybernetics

Research Association of Statistical Sciences, Vol.39

FUKUOKA, JAPAN
2007 


\title{
URYSOHN'S LEMMA IN SCHRÖDER CATEGORIES
}

\author{
By
}

\author{
Yasuo KAWAHARA*
}

\begin{abstract}
A Schröder category extends the category of all binary relations among sets, that is, it realises a relatively huge part of predicate logic. On the other hand Urysohn's lemma asserts that every pair of disjoint closed subsets in a $T_{4}$ topological space can be separated by a continuous function into the reals. Usually the lemma is demonstrated with calculus of elementary set theory. However the structure of this lemma is very interesting from a view point of lattice theory and relational method. This paper gives a relational proof for Urysohn's lemma within Schröder categories.
\end{abstract}

Key Words and Phrases: Binary Relation, Schröder category, $T_{4}$-space, Urysohn's Lemma.

\section{Introduction}

Relational methods (2006) have been developed a number of algebraic theories and applications of (binary) relations in not only mathematics but also computer science, e.g. graph theory (1993), program semantics (1986), (1986), network flows (2006) and so on. Major algebraic frameworks for relations are relation algebras (1941), allegories (1990), Schröder categories and Dedekind categories (1980). Relation algebras due to Tarski founded the modern algebraic system summarising so far study on logic of relations. Allegories serves a foundation for various relational categories including Schröder and Dedekind categories. Schröder categories naturally extend the category of all relations among sets, that is, it realises a relatively huge part of predicate logic. Hom-sets in a Dedekind category are Heyting algebras, instead of boolean algebras in Schröder categories. Though Dedekind categories are weaker than Schröder categories, they give a formal model of fuzzy relations that are important to engineering applications. On the other hand Urysohn's lemma shows that $T_{4}$ topological spaces satisfy the functional separation property and is a crux to prove Urysohn's imbedding theorem. Usually the lemma is demonstrated with a series of calculus in elementary set theory. However the structure of this lemma is very interesting from a view point of lattice theory and relational method. This paper gives a relational proof for Urysohn's lemma within Schröder categories.

We now review the fundamentals on Urysohn's lemma. Let $\left\langle X, \mathcal{O}_{X}\right\rangle$ be a topological space. A subset $U$ of $X$ is called open if $U \in \mathcal{O}_{X}$, and a subset $C$ of $X$ is called closed if its complement $C^{-}$is open. For a subset $S$ of $X$ its closure $S^{\bullet}$ is the least

\footnotetext{
* Department of Informatics, Kyushu University, Fukuoka 819-0395 Japan. kawahara@i.kyushu-u.ac.jp
} 
closed subset of $X$ containing $S$, that is, the intersection of all closed subsets containing $S$.

The fourth separation axiom, so-called $T_{4}$-axiom, for topological spaces was defined as follows:

Definition 1.1 $T_{4}$-AXIom. For every pair of disjoint closed subsets $C$ and $D$ of $X$ there exists a pair of disjoint open subsets $U$ and $V$ of $X$ such that $C \subseteq U$ and $D \subseteq V$.

It is well-known that the $T_{4}$-axiom is equivalent to the following

( $T_{4}^{\prime}$-axiom) For every pair of a closed subset $C$ and an open subset $U$ of $X$ with $C \subseteq U$ there exists an open subsets $V$ of $X$ such that $C \subseteq V \subseteq V^{\bullet} \subseteq U$.

Urysohn's lemma below shows that $T_{4}$ spaces satisfy the functional separation property and leads Urysohn's imbedding theorem that normal spaces with countable basis can be imbedded in the Hilbert cube.

Theorem 1.2 URYsohn's Lemma. Let $X$ be a $T_{4}$-space. For every pair of disjoint closed subsets $C$ and $D$ of $X$ there exists a continuous function $f: X \rightarrow[0,1]$ such that $f(x)=0$ if $x \in C$ and $f(x)=1$ if $x \in D$.

Sketch of Proof. Let $L=[0,1]$ be the unit interval and let

$$
T=\left\{\frac{m}{2^{n}} \mid n, m \in \mathbb{N} \text { and } 0<m<2^{n}\right\}
$$

be a dense subset of $L$ with an injection $j: T \rightarrow L$. Construct a $T$-indexed set $\left\{U_{t} \mid t \in\right.$ $T$ \} of open sets in $X$ such that

$$
\forall s, t \in T . t<s \rightarrow C \subseteq U_{t} \subseteq U_{t}^{\bullet} \subseteq U_{s} \subseteq D^{-} .
$$

Define a function $f: X \rightarrow L$ by

$$
f(x)= \begin{cases}\inf \left\{t \in T \mid x \in U_{t}\right\} & \text { if } x \in \cup_{t \in T} U_{t}, \\ 1 & \text { otherwise. }\end{cases}
$$

It is clear that $0 \leq f(x) \leq 1$ for all $x \in X$, and $f(x)=0$ on $C$, and $f(x)=1$ on $D$. Finally the continuity of $f$ follows from a fact that

$$
\forall a, b \in L . f^{-1}[0, a)=\cup_{t \in T, t<a} U_{t} \wedge f^{-1}(b, 1]=\cup_{t \in T, b<t} U_{t}^{\bullet-} .
$$

The $T$-indexed set $\left\{U_{t} \mid t \in T\right\}$ of open sets in the above proof is regarded as a (binary) relation $\mu \subseteq X \times T$ by $(x, t) \in \mu \leftrightarrow x \in U_{t}$, and another $T$-indexed set $\left\{U_{t}^{\bullet-} \mid\right.$ $t \in T\}$ of open sets corresponds with a relation $\nu \subseteq X \times T$ by $(x, t) \in \nu \leftrightarrow x \in U_{t}^{\bullet-}$. The condition $U_{t} \subseteq U_{t}^{\bullet}$ in $(*)$ is simply written as $\mu \sqsubseteq \nu^{-}$using relational expression. Also from the condition $\forall s, t \in T . t<s \rightarrow U_{t}^{\bullet} \subseteq U_{s}$ we have

$$
\begin{aligned}
(x, t) \in \nu^{-} & \leftrightarrow x \in U_{t}^{\bullet} \\
& \rightarrow \forall s \in T . t<s \rightarrow x \in U_{s} \quad\left\{U_{t}^{\bullet} \subseteq U_{s}\right\} \\
& \leftrightarrow \forall s \in T .(x, s) \in \mu^{-} \rightarrow s \leq t .
\end{aligned}
$$


The last condition will be written as $\nu^{-} \sqsubseteq \mu^{-} \ominus j \xi j^{\sharp}$, where $j, \mu^{-}$and $\ominus$ denote the inclusion map of $T \subseteq L$, the complement of $\mu$ and the residual composition, respectively. This is a motivation of the paper.

This paper is organised as follows. In section 2 we will review the definition and some fundamentals on Schröder categories. In section 3 we will state the definition of topologies and continuous functions in Schröder categories. In section 4 we will demonstrate main results corresponding to Urysohn's lemma in Schröder categories.

\section{Schröder Categories}

In this section we recall the definition of a kind of relation category which we will call Schröder categories (2000), (1993). Schröder categories are Dedekind categories whose hom-sets are complete boolean algebras.

Throughout this paper, a morphism $\alpha$ from an object $X$ into an object $Y$ in a Schröder category (defined below) will be denoted by a half arrow $\alpha: X \rightarrow Y$, and the composition of a morphism $\alpha: X \rightarrow Y$ followed by a morphism $\beta: Y \rightarrow Z$ will be written as $\alpha \beta: X \rightarrow Z$. Also we will denote the identity morphism on $X$ as $\operatorname{id}_{X}$.

Definition 2.1. A Schröder category $\mathcal{S}$ is a category satisfying the following four conditions:

S1. [Complete Boolean Algebra] For all pairs of objects $X$ and $Y$ the hom-set $\mathcal{S}(X, Y)$ consisting of all morphisms of $X$ into $Y$ is a complete Boolean algebra with the least morphism $0_{X Y}$ and the greatest morphism $\nabla_{X Y}$. Its algebraic structure will be denoted by

$$
\mathcal{S}(X, Y)=\left(\mathcal{S}(X, Y), \sqsubseteq, \sqcup, \sqcap,{ }^{-}, 0_{X Y}, \nabla_{X Y}\right),
$$

where $\sqsubseteq, \sqcup, \sqcap$ and ${ }^{-}$denote the inclusion order, the join, the meet and the complement of morphisms, respectively.

S2. [Converse] There is given a converse operation $\sharp: \mathcal{S}(X, Y) \rightarrow \mathcal{S}(Y, X)$. That is, for all morphisms $\alpha, \alpha^{\prime}: X \rightarrow Y, \beta: Y \rightarrow Z$, the following converse laws hold: (a) $(\alpha \beta)^{\sharp}=\beta^{\sharp} \alpha^{\sharp}$, (b) $\left(\alpha^{\sharp}\right)^{\sharp}=\alpha$, (c) If $\alpha \sqsubseteq \alpha^{\prime}$, then $\alpha^{\sharp} \sqsubseteq \alpha^{\prime \sharp}$.

S3. [Dedekind Formula] For all morphisms $\alpha: X \neg Y, \beta: Y \neg Z$ and $\gamma: X \neg Z$ the Dedekind formula $\alpha \beta \sqcap \gamma \sqsubseteq \alpha\left(\beta \sqcap \alpha^{\sharp} \gamma\right)$ holds.

S4. [Zero Relation] The least morphism $0_{X Y}$ is a zero morphism, that is, $\alpha 0_{Y Z}=0_{X Z}$.

A Schröder category is an abstraction of the category of all relations among sets. In what follows, the word relation is a synonym for morphism of Schröder categories.

In a Schröder category $\mathcal{S}$ the converse operation $\sharp: \mathcal{S}(X, Y) \rightarrow \mathcal{S}(Y, X)$ is a $\sqsubseteq$-preserving involutive bijection and so it holds that $0_{X Y}^{\sharp}=0_{Y X}, \nabla_{X Y}^{\sharp}=\nabla_{Y X}$, $\left(\sqcup_{j} \alpha_{j}\right)^{\sharp}=\sqcup_{j} \alpha_{j}^{\sharp},\left(\sqcap_{j} \alpha_{j}\right)^{\sharp}=\sqcap_{j} \alpha_{j}^{\sharp}$ and $\alpha^{-\sharp}=\alpha^{\sharp-}$. Consequently

$$
\mathrm{S} 3^{\sharp} . \alpha \beta \sqcap \gamma \sqsubseteq\left(\alpha \sqcap \gamma \beta^{\sharp}\right) \beta \quad \text { and } \quad \mathrm{S} 4^{\sharp} .0_{X Y} \beta=0_{X Z}
$$

are valid.

An object $I$ of a Schröder category $\mathcal{S}$ is called a (strict) unit if $0_{I I} \neq \operatorname{id}_{I}=\nabla_{I I}$ and $\nabla_{X I} \nabla_{I X}=\nabla_{X X}$ for all objects $X$. A relation $f: X \rightarrow Y$ is called a function, 
denoted by $f: X \rightarrow Y$, if it is univalent $\left(f^{\sharp} f \sqsubseteq \operatorname{id}_{Y}\right)$ and total $\left(\operatorname{id}_{X} \sqsubseteq f f^{\sharp}\right)$. A function $f: X \rightarrow Y$ is called an injection if $f f^{\sharp}=\mathrm{id}_{X}$. The universal relation $\nabla_{X I}: X \rightarrow I$ and the identity relation $\operatorname{id}_{X}: X \rightarrow X$ are functions. An $I$-point $x$ of $X$ is a function $x: I \rightarrow X$.

In Schröder categoreis the residual composition $\alpha \ominus \beta: X \rightarrow Z$ of $\alpha: X \rightarrow Y$ followed by $\beta: Y \rightarrow Z$ can be defined as $\alpha \ominus \beta=\left(\alpha \beta^{-}\right)^{-}$. The residual composition will be frequently used in the paper. For example, the $\operatorname{supremum}$ relation $\sup (\rho, \xi): V \rightarrow X$ is defined by

$$
\sup (\rho, \xi)=(\rho \ominus \xi) \sqcap\left((\rho \ominus \xi) \ominus \xi^{\sharp}\right)
$$

for a pair of relations $\rho: V \rightarrow X$ and $\xi: X \rightarrow X$. The following lemma shows four logical equivalences in Schröder categories.

Lemma 2.2. Let $\alpha: X \rightarrow Y, \beta: Y \rightarrow Z$ and $\gamma: X \rightarrow Z$ be relations in a Schröder category $\mathcal{S}$. Then the following holds.

(a) $\alpha \beta \sqsubseteq \gamma \leftrightarrow \alpha^{\sharp} \gamma^{-} \sqsubseteq \beta^{-} \leftrightarrow \gamma^{-} \beta^{\sharp} \sqsubseteq \alpha^{-}$,

(Schröder equivalence)

(b) $\gamma \sqsubseteq \alpha \ominus \beta \leftrightarrow \alpha^{\sharp} \gamma \sqsubseteq \beta$,

(Residual equivalence)

(c) $\gamma \sqsubseteq \alpha \ominus \beta \leftrightarrow \alpha \sqsubseteq \gamma \ominus \beta^{\sharp}$,

(Galois connection)

Proof. (a) First we will prove the implication $\alpha \beta \sqsubseteq \gamma \rightarrow \alpha^{\sharp} \gamma^{-} \sqsubseteq \beta^{-}$. Assume $\alpha \beta \sqsubseteq \gamma$, which is equivalent to $\alpha \beta \sqcap \gamma^{-}=0_{X Z}$. Then

$$
\begin{aligned}
\alpha^{\sharp} \gamma^{-} \sqcap \beta & \sqsubseteq \alpha^{\sharp}\left(\gamma^{-} \sqcap \alpha \beta\right) & & \{\text { Dedekind Formula S3 } \\
& =\alpha^{\sharp} 0_{X Z} & & \left\{\alpha \beta \sqcap \gamma^{-}=0_{X Z}\right\} \\
& =0_{Y Z}, & & \{\text { Zero Relation S4 }\}
\end{aligned}
$$

which implies $\alpha^{\sharp} \gamma^{-} \sqsubseteq \beta^{-}$. The converse implication $\alpha \beta \sqsubseteq \gamma \rightarrow \alpha^{\sharp} \gamma^{-} \sqsubseteq \beta^{-}$is a variant of the first implication. The proof of another equivalence $\alpha \beta \sqsubseteq \gamma \leftrightarrow \gamma^{-} \beta^{\sharp} \sqsubseteq \alpha^{-}$is analogous.

(b) The residual equivalence is direct from

$$
\begin{aligned}
\gamma \sqsubseteq \alpha \ominus \beta & \leftrightarrow \alpha \beta^{-} \sqsubseteq \gamma^{-} & \text {\{ Complement }\} \\
& \leftrightarrow \alpha^{\sharp} \gamma \sqsubseteq \beta . & \text { \{ Schröder equiv. (a) }\}
\end{aligned}
$$

(c) The Galois connection is clear from the following

$$
\begin{aligned}
\gamma \sqsubseteq \alpha \ominus \beta & \leftrightarrow \alpha^{\sharp} \gamma \sqsubseteq \beta & & \{\text { Residual equiv. (b) }\} \\
& \leftrightarrow \gamma^{\sharp} \alpha \sqsubseteq \beta^{\sharp} & & \{\text { Converse \} } \\
& \leftrightarrow \alpha \sqsubseteq \gamma \ominus \beta^{\sharp} . & & \{\text { Residual equiv. (b) }\}
\end{aligned}
$$

The residual equivalence proved above indicates a fact that a Schröder category is a Dedekind category. The basic properties of Schröder categories are listed in the following proposition, which will be demonstrated in the appendix at the end of the paper. 
Proposition 2.3. Let $\alpha, \alpha^{\prime}: X \rightarrow Y, \beta, \beta^{\prime}: Y \rightarrow Z, \gamma: Z \rightarrow U, \delta: U \rightarrow Z$, $\rho: V \rightarrow X$ and $\xi: X \rightarrow X$ relations in a Schröder category $\mathcal{S}$. Then the following holds.

(a) If $\alpha \sqsubseteq \alpha^{\prime}$ and $\beta \sqsubseteq \beta^{\prime}$ then $\alpha \beta \sqsubseteq \alpha^{\prime} \beta^{\prime}$,

(b) If $\alpha \sqsubseteq \alpha^{\prime}$ and $\beta \sqsubseteq \beta^{\prime}$ then $\alpha^{\prime} \ominus \beta \sqsubseteq \alpha \Theta \beta^{\prime}$,

(c) $\alpha\left(\sqcup_{j} \beta_{j}\right)=\sqcup_{j} \alpha \beta_{j}$ and $\left(\sqcup_{j} \alpha_{j}\right) \beta=\sqcup_{j} \alpha_{j} \beta$,

(d) If $\alpha$ and $\delta$ are univalent then $\alpha\left(\beta \sqcap \beta^{\prime}\right) \delta^{\sharp}=\alpha \beta \delta^{\sharp} \sqcap \alpha \beta^{\prime} \delta^{\sharp}$,

(e) If $\alpha$ is total, $\alpha^{\prime}$ is univalent and $\alpha \sqsubseteq \alpha^{\prime}$ then $\alpha=\alpha^{\prime}$,

(f) $\alpha \ominus(\beta \ominus \gamma)=\alpha \beta \ominus \gamma$ and $(\alpha \ominus \beta) \gamma \sqsubseteq \alpha \ominus \beta \gamma$,

(g) $\alpha \sqsubseteq(\alpha \ominus \beta) \ominus \beta^{\sharp}$,

(h) $\alpha \ominus \beta=\left((\alpha \ominus \beta) \ominus \beta^{\sharp}\right) \ominus \beta$,

(i) If $\alpha$ is a function then $\alpha \ominus \beta=\alpha \beta$ and $\alpha(\beta \ominus \gamma)=\alpha \beta \ominus \gamma$,

(j) If $\beta$ is a function then $\alpha \beta \ominus \gamma=\alpha \ominus \beta \gamma$,

(k) If $\delta$ is a function then $(\alpha \ominus \beta) \delta^{\sharp}=\alpha \ominus \beta \delta^{\sharp}$,

(l) $\sup \left(\rho, \xi^{\sharp}\right)=\sup \left(\rho \ominus \xi^{\sharp}, \xi\right)$,

(m) $\sup (\rho, \xi) \sqsubseteq \rho \ominus \xi \sqsubseteq \sup (\rho, \xi) \ominus \xi$,

(n) If $\xi \xi \sqsubseteq \xi$ and $\sup (\rho, \xi)$ is total then $\sup (\rho, \xi) \xi=\rho \ominus \xi$,

(o) If $f: W \rightarrow V$ is a function then $f \sup (\rho, \xi)=\sup (f \rho, \xi)$,

(p) If $\xi \sqcap \xi^{\sharp} \sqsubseteq \operatorname{id}_{X}$ then $\sup (\rho, \xi)$ is univalent.

(q) If $\alpha$ and $\delta$ are functions then $\left(\alpha \beta \delta^{\sharp}\right)^{-}=\alpha \beta^{-} \delta^{\sharp}$,

(r) $(\alpha \ominus \beta)^{\sharp}=\beta^{-\sharp} \ominus \alpha^{-\sharp}$.

A relation $\xi: L \rightarrow L$ in a Schröder category $\mathcal{S}$ is called an order if it is reflexive $\left(\operatorname{id}_{L} \sqsubseteq \xi\right)$, transitive $(\xi \xi \sqsubseteq \xi)$ and antisymmetric $\left(\xi \sqcap \xi^{\sharp} \sqsubseteq \mathrm{id}_{L}\right)$. Then inclusions $\xi^{-} \xi^{\sharp} \sqsubseteq \xi^{-}$and $\xi^{\sharp} \xi^{-} \sqsubseteq \xi^{-}$hold by applying the Schröder equivalence to $\xi \xi \sqsubseteq \xi$. Also note that $\xi^{\sharp} \ominus \xi=\xi$ iff $\operatorname{id}_{X} \sqsubseteq \xi$ and $\xi \xi \sqsubseteq \xi$. An order $\xi$ is complete if $\sup (\rho, \xi)$ is total (consequently, a function by $2.3(\mathrm{p})$ ) for all relations $\rho: V \rightarrow L$. For each complete order $\xi: L \rightarrow L$ we can define two $I$-points $\perp_{L}$ and $\top_{L}$ by

$$
\perp_{L}=\sup \left(0_{I L}, \xi\right) \quad \text { and } \quad \top_{L}=\sup \left(\nabla_{I L}, \xi\right) .
$$

The next proposition claims the basic property of $I$-points $\perp_{L}$ and $T_{L}$ for complete orders.

Proposition 2.4. Let $\xi: L \rightarrow L$ be a complete order. The the following holds.

(a) $\perp_{L}=\nabla_{I L} \ominus \xi^{\sharp}$ and $\perp_{L} \xi=\nabla_{I L}$, 
(b) $\top_{L}=\nabla_{I L} \ominus \xi$ and $\top_{L} \xi^{\sharp}=\nabla_{I L}$.

Proof. (a) The first identity is direct by $0_{I L} \ominus \xi=\nabla_{I L}$. The second identity simply follows from $\sup \left(0_{I L}, \xi\right) \xi=0_{I L} \ominus \xi=\nabla_{I L}$ using $2.3(\mathrm{n})$.

(b) Note that $\sup \left(\nabla_{I L}, \xi\right)=\sup \left(0_{I L} \ominus \xi^{\sharp}, \xi\right)=\sup \left(0_{I L}, \xi^{\sharp}\right)$ by $2.3(\mathrm{l})$. Hence the statement is a dual of (a).

\section{Topologies}

Let $X$ be an object and $\mathcal{S}(I, X)$ the set of all relations from $I$ into $X$ in a Schröder category $\mathcal{S}$ with a unit $I$. For a subset $\mathcal{X}$ of $\mathcal{S}(I, X)$ we define two subsets $J(\mathcal{X})$ and $M(\mathcal{X})$ of $\mathcal{S}(I, X)$ by

$$
\begin{aligned}
\rho \in J(\mathcal{X}) & \leftrightarrow \quad \rho=\sqcup \mathcal{A} \text { for some subset } \mathcal{A} \subseteq \mathcal{X} \\
\rho \in M(\mathcal{X}) & \leftrightarrow \quad \rho=\sqcap \mathcal{A} \text { for some finite subset } \mathcal{A} \subseteq \mathcal{X}
\end{aligned}
$$

The two operators $J$ and $M$ are closure operators, that is, they are expanding $(\mathcal{X} \subseteq$ $M(\mathcal{X})$ and $\mathcal{X} \subseteq J(\mathcal{X}))$, idempotent $(M M(\mathcal{X})=M(\mathcal{X})$ and $J J(\mathcal{X})=J(\mathcal{X}))$ and monotonic (if $\mathcal{X} \subseteq \mathcal{X}^{\prime}$ then $M(\mathcal{X}) \subseteq M\left(\mathcal{X}^{\prime}\right)$ and $J(\mathcal{X}) \subseteq J\left(\mathcal{X}^{\prime}\right)$ ). Since $\mathcal{S}(I, X)$ is a complete boolean algebra, a distributive law

$$
\left(\sqcup_{j_{1} \in K_{1}} \mu_{j_{1}}\right) \sqcap \cdots \sqcap\left(\sqcup_{j_{n} \in K_{n}} \mu_{j_{n}}\right)=\sqcup_{\left(j_{1}, \ldots, j_{n}\right) \in K_{1} \times \cdots \times K_{n}}\left(\mu_{j_{1}} \sqcap \cdots \sqcap \mu_{j_{n}}\right)
$$

holds and so does the inclusion $M J(\mathcal{X}) \subseteq J M(\mathcal{X})$.

Definition 3.1. A subset $\mathcal{O}$ of $\mathcal{S}(I, X)$ is a topology on $X$ if $J(\mathcal{O})=\mathcal{O}$ and $M(\mathcal{O})=\mathcal{O}$.

A topological object $\langle X, \mathcal{O}\rangle$ is a pair of an object $X$ and a topology $\mathcal{O}$ on $X$. In a topological object $\langle X, \mathcal{O}\rangle$ a relation $\rho: I \rightarrow X$ is called open if $\rho \in \mathcal{O}$, and the complement of an open relation is called closed.

For every subset $\mathcal{X}$ of $\mathcal{S}(I, X), J M(\mathcal{X})$ is always a topology on $X$, because $M J M(\mathcal{X})$ $\subseteq J M M(\mathcal{X})=J M(\mathcal{X})$ and $J J M(\mathcal{X})=J M(\mathcal{X})$

Definition 3.2. Let $\left\langle X, \mathcal{O}_{X}\right\rangle$ and $\left\langle Y, \mathcal{O}_{Y}\right\rangle$ be topological objects.

(a) A function $f:\left\langle X, \mathcal{O}_{X}\right\rangle \rightarrow\left\langle Y, \mathcal{O}_{Y}\right\rangle$ simply denotes a function $f: X \rightarrow Y$,

(b) A function $f:\left\langle X, \mathcal{O}_{X}\right\rangle \rightarrow\left\langle Y, \mathcal{O}_{Y}\right\rangle$ is continuous if $\sigma f^{\sharp} \in \mathcal{O}_{X}$ for all relations $\sigma \in \mathcal{O}_{Y}$.

Now we recall a simpler way to show the continuity of functions with a subbasis of topology.

Proposition 3.3. Let $f: X \rightarrow Y$ be a function and $\mathcal{O}_{Y}=J M(\mathcal{Y})$ for some $\mathcal{Y} \subseteq \mathcal{S}(I, Y)$. Then a function $f:\left\langle X, \mathcal{O}_{X}\right\rangle \rightarrow\left\langle Y, \mathcal{O}_{Y}\right\rangle$ is continuous if $\sigma f^{\sharp} \in \mathcal{O}_{X}$ for all relations $\sigma \in \mathcal{Y}$. 
Proof. First let $\tau \in M(\mathcal{Y})$. Then there is a finite subset $\left\{\nu_{1}, \ldots, \nu_{n}\right\}$ of $\mathcal{Y}$ such that $\tau=\sqcap_{k=1}^{n} \nu_{k}$. It follows from

$$
\tau f^{\sharp}=\left(\sqcap_{k=1}^{n} \nu_{k}\right) f^{\sharp}=\sqcap_{k=1}^{n} \nu_{k} f^{\sharp} \quad\{2.3(\mathrm{~d})\}
$$

that $\tau f^{\sharp} \in \mathcal{O}_{X}$ by the assumption. Next let $\sigma \in \mathcal{O}_{X}=J M(\mathcal{Y})$. Then there is a subset $\left\{\tau_{j}\right\}_{j \in J}$ of $M(\mathcal{Y})$ such that $\sigma=\sqcup_{j \in J} \tau_{j}$. It follows from

$$
\sigma f^{\sharp}=\left(\sqcup_{j \in J} \tau_{j}\right) f^{\sharp}=\sqcup_{j \in J} \tau_{j} f^{\sharp} \quad\{2.3(\mathrm{c})\}
$$

that $\sigma f^{\sharp} \in \mathcal{O}_{X}$ by the first result. Therefore $f$ is continuous.

\section{Main Results} unit $I$ :

In what follows we assume the following setting in a Schröder category $\mathcal{S}$ with a

Assumption A complete order $\xi: L \rightarrow L$, an injection $j: T \rightarrow L$ and a set $I(T)$ of $I$-points $a: I \rightarrow T$ satisfy the following conditions:

(A1) $\xi^{-} j^{\sharp} j \ominus \xi \sqsubseteq \xi$,

(A2) $\sqcup_{a \in I(T)} a^{\sharp} a=\operatorname{id}_{T}$.

(A3) $a j \xi j^{\sharp} b^{\sharp} \in\left\{0_{I I}, \mathrm{id}_{I}\right\}$ for all $a, b \in I(T)$.

The condition (A1) means that $T$ is dense in $L$, and (A2) and (A3) are special point axioms. Note that (A1) is equivalent to (A1 $\left.1^{\sharp}\right) \xi^{\sharp-} j^{\sharp} j \ominus \xi^{\sharp} \sqsubseteq \xi^{\sharp}$ by $2.3(\mathrm{j})$ and (r).

We choose a topology $J M(\mathcal{T})$ on $L$, where

$$
\mathcal{T}=\left\{a j \xi^{-\sharp} \mid a \in I(T)\right\} \cup\left\{a j \xi^{-} \mid a \in I(T)\right\} .
$$

This topology is an analogy to the usual topology on the unit interval $[0,1]$ generated by open intervals with rational ends.

To define topological objects similar to $T_{4}$-spaces we make use of the existence of two relations which correspond to the $T$-indexed sets with the condition $\left(^{*}\right)$ stated in the introduction.

Definition $4.1 T_{4}$-OBJECTs. A topological object $\langle X, \mathcal{O}\rangle$ (in a Schröder category $\mathcal{S}$ ) is called a $T_{4}$-object if for every pair of closed relations $\rho, \sigma: I \rightarrow X$ with $\rho \sqcap \sigma=0_{I X}$ there exists a pair of relations $\mu, \nu: X \rightarrow T$ satisfying the following conditions:

(a) $\rho \mu=0_{I T}$ and $\sigma \nu=0_{I T}$,

(b) $\mu \sqsubseteq \nu^{-} \sqsubseteq \mu^{-} \odot j \xi^{\sharp} j^{\sharp}$,

(c) $a \mu^{\sharp} \in \mathcal{O}$ and $a \nu^{\sharp} \in \mathcal{O}$ for all $a \in I(T)$.

Now we state the main theorem asserting a relational version of Urysohn's lemma for ordinary $T_{4}$-spaces. 
Theorem 4.2 URYsohn's Lemma. Let $\langle X, \mathcal{O}\rangle$ be a $T_{4}$-object. For every pair of closed relations $\rho: I \rightarrow X$ and $\sigma: I \rightarrow X$ with $\rho \sqcap \sigma=0_{I X}$ there exists a continuous function $f:\langle X, \mathcal{O}\rangle \rightarrow\langle L, J M(\mathcal{T})\rangle$ such that $x f=\perp_{L}$ for all $I$-points $x: I \rightarrow X$ with $x \sqsubseteq \rho$ and $y f=\top_{L}$ for all I-points $y: I \rightarrow X$ with $y \sqsubseteq \sigma$.

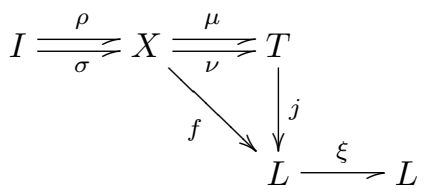

Proof. By the definition 4.1 there exists a pair of relations $\mu, \nu: X \rightarrow T$ satsfying the conditions 4.1 (a), (b) and (c). Define two relations $f, g: X \rightarrow L$ by $f=\sup (\mu j, \xi)$ and $g=\sup \left(\nu j, \xi^{\sharp}\right)$. As the order $\xi$ is complete, $f$ and $g$ are functions. Also note that $f \xi=\mu j \ominus \xi$ and $g \xi^{\sharp}=\nu j \ominus \xi^{\sharp}$ by $2.3(\mathrm{n})$. Now the identity $f=g$ will follow from (U1) - (U4) below:

(U1) $f \sqsubseteq g \xi^{\sharp}:$ By 4.1(b) we have

$$
\mu \sqsubseteq \nu^{-} \sqsubseteq \mu^{-} \ominus j \xi^{\sharp} j^{\sharp} \sqsubseteq \nu \ominus j \xi^{\sharp} j^{\sharp}=g \xi^{\sharp} j^{\sharp},
$$

which is equivalent to $\mu j \sqsubseteq g \xi^{\sharp}$, because $j$ is a function. Hence

$$
\begin{aligned}
& f \sqsubseteq(\mu j \ominus \xi) \ominus \xi^{\sharp} \quad\{f=\sup (\mu j, \xi)\} \\
& \sqsubseteq\left(g \xi^{\sharp} \ominus \xi\right) \ominus \xi^{\sharp} \quad\left\{\mu j \sqsubseteq g \xi^{\sharp}, 2.3(\mathrm{~b})\right\} \\
& =g \xi^{\sharp} . \quad\left\{g \text { : function and } \xi^{\sharp} \ominus \xi=\xi\right\}
\end{aligned}
$$

(U2) $g \xi^{-} j^{\sharp} \sqsubseteq \mu$ :

$$
\begin{aligned}
& \mu^{-} \sqsubseteq \nu^{-} \ominus j \xi j^{\sharp} \quad\{4.1(\mathrm{~b}), \text { Galois conn. }\} \\
& \sqsubseteq g \xi^{-} j^{\sharp} \odot j \xi j^{\sharp} \quad\left\{\nu \sqsubseteq g \xi j^{\sharp} \text { by } g \sqsubseteq \nu j \odot \xi^{\sharp}\right\} \\
& =g\left(\xi^{-} j^{\sharp} j \Theta \xi\right) j^{\sharp} \quad\{g, j \text { : function }\} \\
& \sqsubseteq g \xi j^{\sharp} . \quad\{(\mathrm{A} 1)\}
\end{aligned}
$$

Thus taking complements of both sides we have $g \xi^{-} j^{\sharp} \sqsubseteq \mu$.

(U3) $f \sqsubseteq g \xi$ :

$$
\begin{aligned}
& f \sqsubseteq \mu j \ominus \xi \quad\{f=\sup (\mu j, \xi)\} \\
& \sqsubseteq g \xi^{-} j^{\sharp} j \ominus \xi \quad\{(\mathrm{U} 2)\} \\
& =g\left(\xi^{-} j^{\sharp} j \odot \xi\right) \quad\{g \text { : function }\} \\
& \sqsubseteq g \xi . \quad\{(\mathrm{A} 1)\}
\end{aligned}
$$

(U4) $f=g$ : By (U1), (U3) and the antisymmetry of $\xi$ it holds that

$$
f \sqsubseteq g \xi \sqcap g \xi^{\sharp}=g\left(\xi \sqcap \xi^{\sharp}\right) \sqsubseteq g .
$$

Therefore we have $f=g$ by $2.3(\mathrm{e})$. Next we will show that $f:\langle X, \mathcal{O}\rangle \rightarrow\langle L, J M(\mathcal{T})\rangle$ is continuous. By 3.3 it suffices to see that $a j \xi^{-\sharp} f^{\sharp} \in \mathcal{O}$ and $a j \xi^{-} f^{\sharp} \in \mathcal{O}$ for all $a \in I(T)$. Let $a \in I(T)$ and set $I_{a}=\left\{b \in I(T) \mid a j \xi^{\sharp} j^{\sharp} b^{\sharp}=0_{I I}\right\}$. Then

$$
\begin{aligned}
& a j \xi^{-\sharp} f^{\sharp}=a j(f \xi)^{-\sharp} \quad\left\{(f \xi)^{-}=f \xi^{-}, 2.3(\mathrm{q})\right\} \\
& =a j \xi^{-\sharp} j^{\sharp} \mu^{\sharp} \quad\left\{f \xi=\mu j \ominus \xi=\left(\mu j \xi^{-}\right)^{-}\right\} \\
& =\sqcup_{b \in I(T)} a j \xi^{-\sharp} j^{\sharp} b^{\sharp} b \mu^{\sharp} \quad\left\{(\mathrm{A} 2) \sqcup_{b \in I(T)} b^{\sharp} b=\mathrm{id}_{T}\right\} \\
& =\sqcup_{b \in I_{a}} b \mu^{\sharp} . \quad\left\{b \in I_{a} \leftrightarrow a j \xi^{-\sharp} j^{\sharp} b^{\sharp}=\operatorname{id}_{I},(\mathrm{~A} 3)\right\}
\end{aligned}
$$


Hence $a j \xi^{-\sharp} f^{\sharp} \in \mathcal{O}$ by the definition of topologies. Analogously $a j \xi^{-} f^{\sharp} \in \mathcal{O}$ follows from

$$
\begin{aligned}
a j \xi^{-} f^{\sharp} & =a j\left(f \xi^{\sharp}\right)^{-\sharp} & & \left\{(f \xi)^{-}=f \xi^{-}, 2.3(\mathrm{q})\right\} \\
& =a j \xi^{-} j^{\sharp} \nu^{\sharp} & & \left\{f \xi^{\sharp}=\nu j \Theta \xi^{\sharp}=\left(\nu j \xi^{-}\right)^{-}\right\} \\
& =\sqcup_{b \in I(T)} a j \xi^{-} j^{\sharp} b^{\sharp} b \nu^{\sharp} & & \left\{(\mathrm{A} 2) \sqcup_{b \in I(T)} b^{\sharp} b=\mathrm{id}_{T}\right\} \\
& =\sqcup_{b \in I_{a}^{\sharp}} b \nu^{\sharp}, & & \left\{b \in I_{a}^{\sharp} a j \xi^{-} j^{\sharp} b^{\sharp}=\mathrm{id}_{I},(\mathrm{~A} 3)\right\}
\end{aligned}
$$

where $I_{a}^{\sharp}=\left\{b \in I(T) \mid a j \xi j^{\sharp} b^{\sharp}=0_{I I}\right\}$. Finally let $x, y: I \rightarrow X$ be $I$-points with $x \sqsubseteq \rho$ and $y \sqsubseteq \sigma$, respectively. Then

$$
\begin{array}{rlrl}
x f & =\sup (x \mu j, \xi) & \{x: \text { function, } 2.3(\mathrm{o})\} \\
& =\sup \left(0_{I L}, \xi\right) \quad\left\{x \mu \sqsubseteq \rho \mu=0_{I T}\right\} \\
& =\perp_{L},
\end{array}
$$

and

$$
\begin{aligned}
y f & =\sup \left(y \nu j, \xi^{\sharp}\right) & & \{f=g \text { and } y \text { : function, } 2.3(\mathrm{o})\} \\
& =\sup \left(0_{I L}, \xi^{\sharp}\right) & & \left\{y \nu \sqsubseteq \sigma \nu=0_{I T}\right\} \\
& =\top_{L} . & & \{2.4\}
\end{aligned}
$$

This completes the proof of the theorem.

The topology $J M(\mathcal{T})$ on $L$ coincides with a more natural topology under stronger conditions.

Proposition 4.3. Let $I(L)$ be a set of $I$-points $x: I \rightarrow L$ and set $\mathcal{T}_{0}=\left\{x \xi^{-} \mid\right.$ $x \in I(L)\} \cup\left\{x \xi^{-\sharp} \mid x \in I(L)\right\}$. If (B1) aj $\in I(L)$ for all $a \in I(T)$ and (B2) $x \xi j^{\sharp} a^{\sharp} \in$ $\left\{0_{I I}, \mathrm{id}_{I}\right\}$ and $x \xi^{\sharp} j^{\sharp} a^{\sharp} \in\left\{0_{I I}, \mathrm{id}_{I}\right\}$ for all $x \in I(L)$ and $a \in I(T)$, then $J M\left(\mathcal{T}_{0}\right)=$ $J M(\mathcal{T})$.

Proof. By (B1) it is trivial that $\mathcal{T} \subseteq \mathcal{T}_{0}$. We will see $\mathcal{T}_{0} \subseteq J(\mathcal{T})$. Let $x \in I(L)$ and set $I_{x}=\left\{a \in I(T) \mid x \xi j^{\sharp} a^{\sharp}=0_{I I}\right\}$. Then it holds that

$$
\begin{aligned}
x \xi^{-} & =x \xi^{-} j^{\sharp} j \xi^{-} & & \left\{(\mathrm{A} 1) \xi^{-}=\xi^{-} j^{\sharp} j \xi^{-}\right\} \\
& =\sqcup_{a \in I(T)} x \xi^{-} j^{\sharp} a^{\sharp} a j \xi^{-} & & \left\{(\mathrm{A} 2) \sqcup_{a \in I(T)} a^{\sharp} a=\mathrm{id}_{T}\right\} \\
& =\sqcup_{a \in I_{x}} a j \xi^{-}, & & \left\{a \in I_{x} \leftrightarrow x \xi^{-} j^{\sharp} a^{\sharp}=\mathrm{id}_{I},(\mathrm{~B} 2)\right\}
\end{aligned}
$$

which proves $x \xi^{-} \in J(\mathcal{T})$. Analogously $x \xi^{-\sharp} \in J(\mathcal{T})$ follows from

$$
\begin{aligned}
x \xi^{-\sharp} & =x \xi^{-\sharp} j^{\sharp} j \xi^{-\sharp} & & \left\{\left(\mathrm{A} 1^{\sharp}\right) \xi^{-\sharp}=\xi^{-\sharp} j^{\sharp} j \xi^{-\sharp}\right\} \\
& =\sqcup_{a \in I(T)} x \xi^{-\sharp} j^{\sharp} a^{\sharp} a j \xi^{-\sharp} & & \left\{(\mathrm{A} 2) \sqcup_{a \in I(T)} a^{\sharp} a=\mathrm{id}_{T}\right\} \\
& =\sqcup_{a \in I_{x}^{\sharp}} a j \xi^{-\sharp}, & & \left\{a \in I_{x}^{\sharp} \leftrightarrow x \xi^{-\sharp} j^{\sharp} a^{\sharp}=\mathrm{id}_{I},(\mathrm{~B} 2)\right\}
\end{aligned}
$$

where $I_{x}^{\sharp}=\left\{a \in I(T) \mid x \xi^{\sharp} j^{\sharp} a^{\sharp}=0_{I I}\right\}$. Hence we have $J M(\mathcal{T}) \subseteq J M\left(\mathcal{T}_{0}\right) \subseteq J M J(\mathcal{T}) \subseteq$ $J M(\mathcal{T})$.

Remark. The conditions (A3) and (B2) are trivial if there is no relations in $\mathcal{S}(I, I)$ except for $0_{I I}$ and $\mathrm{id}_{I}$. 


\section{Conclusion}

The paper demonstrated Urysohn's Lemma in Schröder categories by regarding a series of open subsets as a binary relation. The results of the paper shows the capability of relational methods in application to mathematical analysis. On the other hand the author encountered some difficulties to apply theory of relations to even a part of general topology, because usual mathematics unconsciously uses the essential property of points. The notion of $I$-points contains not only crisp (or standard) $I$-points but also non crisp ones. To avoid the disadvantage caused by the fact, the author assumed the sets $I(T)$ and $I(X)$ with some strong properties.

\section{Acknowledgement}

The author is grateful to anonymous referee for his helpful comments and suggestions.

\section{References}

Bergharmmer, R. and Zierer, H. (1986). Relational algebraic semantics of determininstic and nondeterministic programs, Theor. Comput. Sci. 43, 123-147.

Freyd, P. and Scedrov, A. (1990). Categories, allegories, North-Holland, Amsterdam.

Hoare, C. A. R and Jifeng, H. (1986). The weakest prespecification, Part 1. Fund. Inform. 9(4), 51-84.

Kawahara, Y. (2000). Theory of Dedekind categories, unpublished manuscript.

Kawahara, Y. (2006). On the cardinality of relations, Lecture Notes in Computer Science 4136, 251-265.

MacLane, S. (1971). Categories for the working mathematician, Springer-Verlag.

Olivier, J. P. and Serrato, D. (1980). Catégories de Dedekind. Morphismes dans les Catégories de Schröder, C. R. Acad. Sci. Paris 260, 939-941.

Schmidt, G. and Ströhlein, T. (1993). Relations and graphs - Discrete Mathematics for Computer Science -, Springer-Verlag, Berlin.

Schmidt, R. (Ed.) (2006). Relations and Kleene algebra in computer science, Lecture Notes in Computer Science 4136.

Tarski, A. (1941). On the calculus of relations, J. Symbolic Logic 6, 73-89.

\section{Appendix}

In the appendix we give the proof of Proposition 2.3.

(a) Assume $\alpha \sqsubseteq \alpha^{\prime}$ and $\beta \sqsubseteq \beta^{\prime}$. Then

$$
\begin{aligned}
\beta & \sqsubseteq \beta^{\prime} \\
& \sqsubseteq \alpha^{\sharp} \ominus \alpha \beta^{\prime}, \quad\left\{\alpha \beta^{\prime} \sqsubseteq \alpha \beta^{\prime} \text { and } 2.2(\mathrm{~b})\right\}
\end{aligned}
$$


which proves $\alpha \beta \sqsubseteq \alpha \beta^{\prime}$ again by 2.2(b). Another inclusion $\alpha \beta \sqsubseteq \alpha^{\prime} \beta$ is obtained by applying the converse.

(b) Assume $\alpha \sqsubseteq \alpha^{\prime}$ and $\beta \sqsubseteq \beta^{\prime}$. Then

$$
\begin{aligned}
\alpha^{\sharp}\left(\alpha^{\prime} \ominus \beta\right) & \sqsubseteq \alpha^{\prime \sharp}\left(\alpha^{\prime} \ominus \beta\right) & & \left\{\alpha \sqsubseteq \alpha^{\prime}, \mathrm{S} 2(\mathrm{c}) \text { and }(\mathrm{a})\right\} \\
& \sqsubseteq \beta & & \left\{\alpha^{\prime} \ominus \beta \sqsubseteq \alpha^{\prime} \ominus \beta \text { and } 2.2(\mathrm{~b})\right\} \\
& \sqsubseteq \beta^{\prime}, & &
\end{aligned}
$$

which shows $\alpha^{\prime} \Theta \beta \sqsubseteq \alpha \Theta \beta^{\prime}$ again by $2.2(\mathrm{~b})$.

(c) The first identity follows from

$$
\begin{array}{rlrl}
\alpha\left(\sqcup_{j} \beta_{j}\right) \sqsubseteq \gamma & \leftrightarrow \sqcup_{j} \beta_{j} \sqsubseteq \alpha^{\sharp} \ominus \gamma \quad\{\text { Res. equiv. 2.2(b) } \\
& \leftrightarrow \forall j . \beta_{j} \sqsubseteq \alpha^{\sharp} \ominus \gamma & \\
& \leftrightarrow \forall j . \alpha \beta_{j} \sqsubseteq \gamma & \text { \{ Res. equiv. 2.2(b) }\} \\
& \leftrightarrow \sqcup_{j} \alpha \beta_{j} \sqsubseteq \gamma . &
\end{array}
$$

The second identity $\left(\sqcup_{j} \alpha_{j}\right) \beta=\sqcup_{j} \alpha_{j} \beta$ is obtained by applying the converse.

(d) It follows from

$$
\begin{array}{rlll}
f\left(\alpha \sqcap \alpha^{\prime}\right) g^{\sharp} & \sqsubseteq f \alpha g^{\sharp} \sqcap f \alpha^{\prime} g^{\sharp} & & \{(\mathrm{a})\} \\
& \sqsubseteq f\left(\alpha \sqcap f^{\sharp} f \alpha^{\prime} g^{\sharp} g\right) g^{\sharp} & & \text { (Dedekind formula S3 and S3 } \left.3^{\sharp}\right\} \\
& \sqsubseteq f\left(\alpha \sqcap \alpha^{\prime}\right) g^{\sharp} . & & \{f, g: \text { univalent and (a) }\}
\end{array}
$$

(e) Assume that $\alpha$ is total, $\alpha^{\prime}$ is univalent and $\alpha \sqsubseteq \alpha^{\prime}$. Then

$$
\begin{aligned}
& \alpha^{\prime} \sqsubseteq \alpha \alpha^{\sharp} \alpha^{\prime} \quad\{\alpha: \text { total }\} \\
& \begin{array}{ll}
\sqsubseteq \alpha \alpha^{\prime \sharp} \alpha^{\prime} & \left\{\alpha \sqsubseteq \alpha^{\prime},(\mathrm{a}), \mathrm{S} 2(\mathrm{c})\right\} \\
\sqsubseteq \alpha, & \left\{\alpha^{\prime}: \text { univalent }\right\}
\end{array}
\end{aligned}
$$

which proves $\alpha=\alpha^{\prime}$.

(f) The first identity follows from the following equivalences.

$$
\begin{aligned}
\eta \sqsubseteq \alpha \ominus(\beta \ominus \gamma) & \leftrightarrow \alpha^{\sharp} \eta \sqsubseteq \beta \ominus \gamma & \text { \{ Res. equiv. 2.2(b) }\} \\
& \leftrightarrow \beta^{\sharp} \alpha^{\sharp} \eta \sqsubseteq \gamma & \text { \{ Res. equiv. 2.2(b) }\} \\
& \leftrightarrow \eta \sqsubseteq \alpha \beta \ominus \gamma . & \text { \{ Res. equiv. 2.2(b) }\}
\end{aligned}
$$

The second inclusion is direct from

$$
\alpha^{\sharp}(\alpha \ominus \beta) \gamma \sqsubseteq \beta \gamma . \quad\left\{\alpha^{\sharp}(\alpha \ominus \beta) \sqsubseteq \beta\right\}
$$

(g) It is simply obtained by applying the Galois connection 2.2(c) to an inclusion $\alpha \Theta \beta \sqsubseteq$ $\alpha \ominus \beta$.

(h) An inclusion $\alpha \ominus \beta \sqsubseteq\left((\alpha \ominus \beta) \ominus \beta^{\sharp}\right) \ominus \beta$ is a corollary of (g). The converse inclusion follows from (g) and (b).

(i) Let $\alpha$ be a function. Then

$$
\begin{aligned}
& \alpha \ominus \beta \sqsubseteq \alpha \alpha^{\sharp}(\alpha \odot \beta) \quad\{\alpha \text { : total }\} \\
& \sqsubseteq \alpha \beta \quad\left\{\alpha^{\sharp}(\alpha \ominus \beta) \sqsubseteq \beta,\right\} \\
& \sqsubseteq \alpha \odot \alpha^{\sharp} \alpha \beta \quad\left\{\alpha^{\sharp} \alpha \beta \sqsubseteq \alpha^{\sharp} \alpha \beta\right\} \\
& \sqsubseteq \alpha \ominus \beta . \quad\{\alpha \text { : univalent, (b) }\}
\end{aligned}
$$


The second identity holds from

$$
\alpha(\beta \ominus \gamma)=\alpha \ominus(\beta \ominus \gamma)=\alpha \beta \ominus \gamma
$$

using the first identity and (f).

(j) Let $\beta$ be a function. Then $\alpha \beta \ominus \gamma=\alpha \ominus(\beta \ominus \gamma)=\alpha \ominus \beta \gamma$ by (f) and (i).

(k) Let $\delta$ be a function. Then

$$
\begin{array}{rlll}
(\alpha \Theta \beta) \delta^{\sharp} & \sqsubseteq \alpha \Theta \beta \delta^{\sharp} & & \{(\mathrm{f})\} \\
& \sqsubseteq\left(\alpha \Theta \beta \delta^{\sharp}\right) \delta \delta^{\sharp} & \{\delta: \text { total }\} \\
& \sqsubseteq \alpha \Theta \beta \delta^{\sharp} \delta \delta^{\sharp} & & \{(\mathrm{f})\} \\
& =\alpha \Theta \beta \delta^{\sharp} . & & \{\delta: \text { univalent }\}
\end{array}
$$

(1)

$$
\begin{aligned}
\sup \left(\rho \ominus \xi^{\sharp}, \xi\right) & =\left(\left(\rho \ominus \xi^{\sharp}\right) \ominus \xi\right) \sqcap\left(\left(\left(\rho \ominus \xi^{\sharp}\right) \ominus \xi\right) \ominus \xi^{\sharp}\right) \\
& =\left(\left(\rho \ominus \xi^{\sharp}\right) \ominus \xi\right) \sqcap\left(\rho \ominus \xi^{\sharp}\right) \\
& =\sup \left(\rho, \xi^{\sharp}\right) .
\end{aligned}
$$

(m) The first inclusion is trivial. The second inclusion is deduced by applying the Galois connection to the trivial inclusion $\sup (\rho, \xi) \sqsubseteq(\rho \ominus \xi) \ominus \xi^{\sharp}$.

(n) Assume that $\sup (\rho, \xi)$ is total and $\xi \xi \sqsubseteq \xi$. Then

$$
\begin{array}{rlll}
\sup (\rho, \xi) \xi & \sqsubseteq \rho \ominus \xi & & \{(\mathrm{f}) \text { and } \xi \xi \sqsubseteq \xi\} \\
& \sqsubseteq \sup (\rho, \xi) \ominus \xi & & \{(\mathrm{l})\} \\
& \sqsubseteq \sup (\rho, \xi) \xi . & & \{\sup (\rho, \xi): \text { total }\}
\end{array}
$$

(o) Let $f$ be a function. Then

$$
\begin{aligned}
f \sup (\rho, \xi) & =f\left((\rho \ominus \xi) \sqcap\left((\rho \ominus \xi) \ominus \xi^{\sharp}\right)\right) & \\
& =f(\rho \ominus \xi) \sqcap f\left((\rho \ominus \xi) \ominus \xi^{\sharp}\right) & \{(\mathrm{d})\} \\
& =(f \rho \ominus \xi) \sqcap\left((f \rho \ominus \xi) \ominus \xi^{\sharp}\right) & \{(\mathrm{i})\} \\
& =\sup (f \rho, \xi) . &
\end{aligned}
$$

(p) Let $\xi \sqcap \xi \sqsubseteq \operatorname{id}_{X}$ and set $\tau=\rho \ominus \xi$. Then the univalency of $\sup (\rho, \xi)$ follows from

$$
\begin{array}{rlll}
(\sup (\rho, \xi))^{\sharp} \sup (\rho, \xi) & =\left(\tau \sqcap\left(\tau \ominus \xi^{\sharp}\right)\right)^{\sharp}\left(\tau \sqcap\left(\tau \ominus \xi^{\sharp}\right)\right) & \\
& \sqsubseteq \tau^{\sharp}\left(\tau \ominus \xi^{\sharp}\right) \sqcap\left(\tau \ominus \xi^{\sharp}\right)^{\sharp} \tau & \\
& \sqsubseteq \xi^{\sharp} \sqcap \xi^{\sharp} & & \left\{\tau^{\sharp}\left(\tau \ominus \xi^{\sharp}\right) \sqsubseteq \xi^{\sharp}\right. \\
& \sqsubseteq \operatorname{id}_{X} . & & \left\{\xi \sqcap \xi^{\sharp} \sqsubseteq \operatorname{id}_{X}\right\}
\end{array}
$$

(q) Let $\alpha$ and $\delta$ be functions. Then

$$
\begin{aligned}
\alpha \beta \delta^{\sharp} \sqcap \alpha \beta^{-} \delta^{\sharp} & =\alpha\left(\beta \sqcap \beta^{-}\right) \delta^{\sharp} & & \{(\mathrm{d})\} \\
& =0_{X U}, & & \left\{\beta \sqcap \beta^{-}=0_{Y Z}, \mathrm{~S} 4\right\}
\end{aligned}
$$

and

$$
\begin{array}{rlll}
\nabla_{X U} & \sqsubseteq \alpha \alpha^{\sharp} \nabla_{X U} \delta \delta^{\sharp} & \{\alpha, \delta: \text { total }\} \\
& \sqsubseteq \alpha \nabla_{Y Z^{\prime} \delta^{\sharp}} & \left\{\alpha^{\sharp} \nabla_{X U} \delta \sqsubseteq \nabla_{Y Z}\right\} \\
=\alpha\left(\beta \sqcup \beta^{-}\right) \delta^{\sharp} & \left\{\nabla_{Y Z}=\beta \sqcup \beta^{-}\right\} \\
=\alpha \beta \delta^{\sharp} \sqcup \alpha \beta^{-} \delta^{\sharp} . & \{(\mathrm{c})\}
\end{array}
$$


(r)

$$
\begin{aligned}
(\alpha \ominus \beta)^{\sharp} & =\left(\alpha \beta^{-}\right)^{-\sharp} \\
& =\left(\alpha \beta^{-}\right)^{\sharp-} \\
& =\left(\beta^{-} \alpha^{-\sharp-}\right)^{-} \quad\left\{\alpha^{-\sharp}=\alpha^{\sharp-} \text { and S2(a) }\right\} \\
& =\beta^{-\sharp} \odot \alpha^{-\sharp} .
\end{aligned}
$$

Received April 24, 2007

Revised August 28, 2007 